\section{A P P E N D I X \\ Notes on the Analysis}

The data consist of the reported number of needlestick incidents among intensive care unit (ICU) nurses, the number of ICU nurses, and the number of hours they worked for three successive 30-day periods before and after implementing a new infection control strategy at 26 Canadian hospitals. Preliminary examination revealed that one hospital reported that its staff worked more than 100,000 hours per month. With only about 70 staff members, this works out to more than 50 hours per day, so I omitted the results from the analysis altogether. Results from the remaining hospitals were combined into two 3-month periods before and after the change.

Three of the 26 hospitals accounted for the bulk of all the needlestick injuries. One went from 24 injuries before to one after; one went from 21 before to 26 after; and one went from seven before to zero after. No other hospital had more than five incidents in either period (most were one or zero), nor a comparable rate change. Because these hospitals were so different from the rest and could bias the results, I omitted them from the data and consider them special cases. Note that, taken together, the improvement for these three is enormous, from 52 incidents before to 27 after. The significance of the hospital showing an increase is impossible to judge, of course, without specific information about what happened there.

Of the 22 remaining hospitals, only nine had complete data available on the number of hours worked. Although incidence per hour is the desirable indicator, nine hospitals were too few for reliable analysis. Given the nature of the data gathering, the likelihood of bias was great, and, in any case, the small sample size would produce a uselessly wide confidence interval (the power was too low). So I used incidence per nurse for the 22 hospitals instead. The remaining 22 percentage differences in this rate comprised the data that were analyzed. Figure 1 is a stem-and-leaf display of these differences.

\title{
Diphtheria Epidemic in Russia Related to High Rates of Adult Susceptibility
}

\section{by Gina Pugliese, RN, MS Medical News Editor}

Russia has experienced a resurgence of diphtheria after 30 years of control. Since 1989, reported cases have doubled each year. In 1993, more than 10,000 cases and 300 deaths were reported.

To assess factors contributing to this epidemic, researchers from the Centers for Disease Control and Prevention (CDC) reviewed available surveillance data on disease and vaccine coverage. To evaluate the clinical efficacy of diphtheria vaccine (DV), the researchers analyzed the vaccination status of 207 Muscovites aged $<15$ years, who were diphtheria cases in 1991 and 1992, as compared with matched controls. Multilocus enzyme electrophoresis (MEE) of 50 diphtheria isolates from different regions of Russia were performed.

Although cases were reported from all regions of Russia, 1,609 (23\%) of 6,979 cases reported from 1990 to 1992 were from Moscow, and 1,071 (15\%) were from Saint Petersburg. More than $70 \%$ of cases are among adults, a group not previously targeted for routine vaccination, and $11 \%$ are among children $<7$ years of age. Three or more doses of DV were $95 \%$ effective in preventing clinical diphtheria among children. Among study controls, coverage with three doses of DV was only $40 \%$ by 1 year of age, but was $90 \%$ by age 5. MEE analysis demonstrated the emergence of a single epidemic clone associ- ated with the outbreak; 10 of 13 isolates in 1993 were of this clone, compared with only 2 of 15 isolates from 1986 through 1990.

The authors concluded that the epidemic may be due to the introduction of pathogenic diphtheria into a population with high rates of adult susceptibility. Neither low vaccine effectiveness nor low infant vaccination coverage appear to be major contributors. Control is likely to require extensive vaccination of adults in urban areas.

FROM: Hardy IR, Monisov A, Filatov N, et al. Major epidemic of diphtheria, Russia. Presented at the 43rd Annual Epidemic Intelligence Service Conference in Atlanta; April 18-22, 1994; Atlanta, Georgia. 